\title{
ROBAST 3
}

\section{Akira Okumura ${ }^{a, b, *}$}

${ }^{a}$ Institute for Space-Earth Environmental Research, Nagoya University,

Furo-cho, Chikusa-ku, Nagoya 464-8601, Japan

${ }^{b}$ Kobayashi-Maskawa Institute for the Origin of Particles and the Universe, Nagoya University Furo-cho, Chikusa-ku, Nagoya 464-8602, Japan

E-mail: oxon@mac.com

The ROOT-based simulator for ray tracing (ROBAST) is an open-source C++ library to simulate various types of cosmic-ray (CR) and gamma-ray telescopes. It is used in multiple purposes in all the different telescope designs of the Cherenkov Telescope Array and is a promising tool for use in other future projects in optics simulations. We have released ROBAST 3 that supports interference calculations in multilayer coating in addition to geometrical optics. Here, we present its new functionality and several recent applications in future CR telescopes.

$37^{\text {th }}$ International Cosmic Ray Conference (ICRC 2021)

July 12 th - 23rd, 2021

Online - Berlin, Germany

${ }^{*}$ Presenter 


\section{Introduction}

The ROOT-based simulator for ray tracing (ROBAST) ${ }^{12}$ is an open-source C++ library [1], used for non-sequential ray-tracing simulations in several cosmic-ray (CR) telescope projects. Its initial development started in 2007 when it was used to simulate a modified Baker-Nunn optical system [2] that was composed of segmented spherical mirrors, three aspherical lenses, telescope frame, and many camera components intricately obscuring incident photons. Calculating the effective area of this complex optical system or embedding it in a Monte Carlo (MC) simulation was difficult, more so, no open-source software suitable for similar CR telescopes existed. ROBAST development aims to provide a ray-tracing simulation library for the CR physics community, which is freely available, equipped with necessary functions for CR telescopes, easily used in a common programming language $(\mathrm{C}++)$, and well debugged in multiple $\mathrm{CR}$ telescope projects.

Utilizing the ROOT geometry library and its particle tracking engine [3], complex CR telescopes are easily built in ROBAST, allowing users to simulate mixed reflections, refraction, and scattering of individual photons. Added to the pre-defined ROOT primitive geometry classes, ROBAST has its own geometry classes (shapes) for simulating aspherical surfaces and light concentrators [4], for modeling most CR telescopes without requiring users to implement a new geometry class.

The earliest applications of ROBAST after the Baker-Nunn optics simulation are the segmented parabolic telescope, Schwarzschild-Couder telescopes, and hexagonal light concentrators in the Cherenkov Telescope Array (CTA) [5, 6]. ROBAST not only simulates the point spread functions (PSFs) and effective areas of CTA telescopes, it is also used for mirror alignment tolerance analyses, sunlight avoidance calculations, and optimization of light-concentrator designs in CTA [1, 4]. As of 2021, ROBAST is used for all the telescope subprojects in CTA; the Large-sized Telescopes (LSTs, a segmented $23 \mathrm{~m}$ parabolic system), two Medium-sized Telescopes (MSTs, $12 \mathrm{~m}$ DaviesCotton and $10 \mathrm{~m}$ Schwarzschild-Couder systems), and the Small-sized Telescopes (SSTs, a $4 \mathrm{~m}$ Schwarzschild-Couder system), for various purposes.

Optical simulations in gamma-ray and CR telescopes usually employ geometrical optics (ray tracing) because the mirror roughness in a macro scale is much larger than the Cherenkov and fluorescence wavelengths and their PSF sizes are wider than the diffraction limit by several orders of magnitude. However, multilayer coating, requiring interference calculation, is frequently applied on mirror surfaces to protect the specular aluminum layer and enhance the reflectance in 300 $500 \mathrm{~nm}$. In multilayer coating, the angular and wavelength dependence of mirror reflectance are not negligible when the number of layers is large, thus they are often pre-calculated and the lookup tables are inputted into a geometrical ray-tracing simulation in CR telescopes. For example, this approach is employed in sim_telarray, the standard array simulation MC software in H.E.S.S. and CTA [7]. However, using separate simulation tools for multilayer and ray-tracing simulations is not suitable for some simulation studies, such as the simultaneous optimization of a specular lightconcentrator shape and its surface coating design. Additionally, silicon photomultipliers (SiPMs) show strong wavelength dependence in photon detection efficiency (PDE) curves due to $\mathrm{SiO}_{2}$ and SiN layers when the surface resin coating is removed to suppress their optical crosstalk $[8,9]$. To

${ }^{1}$ https://robast.github.io

${ }^{2}$ https: //github. com/ROBAST 
simulate these quantum effects within a single simulation library, we implemented new ROBAST functionality to simulate multilayer coating in its latest major release (ROBAST $3^{3}$ ).

This study reports the new functionality for multilayer simulations in ROBAST 3 and presents recent applications of ROBAST in different types of CR telescopes.

\section{Multilayer Support in ROBAST 3}

\subsection{Implementation}

Multilayer simulation needs in CR telescopes are often found in mirror development because it needs high (> 90\%) reflectance in the UV-blue region for Cherenkov and fluorescence photons and low reflectance (high absorptance) in the red region to reduce the night-sky background. Most coating companies use commercial and proprietary software in the material selection and thickness optimization phase of layer design in multilayer coatings. However, the interference of multilayer coatings can be calculated using basic knowledge of plane waves. Also, several open-source libraries for multilayer simulations are available.

We did not reinvent a wheel in developing ROBAST 3, rather we re-implemented an existing Python package, $\mathrm{tmm}^{4}$, in $\mathrm{C}++$. The tmm package and its calculation methods are used in several studies, and the details are described in [10].

\subsection{Bandpass Multilayer Coating}

Fig 1 shows an example of a combined multilayer and geometrical ray-tracing simulation of hexagonal light concentrator (Winston cone) using ROBAST 3. In this example, we assumed that nine thin layers composed of $\mathrm{SiO}_{2}, \mathrm{Ta}_{2} \mathrm{O}_{5}, \mathrm{Al}$, and $\mathrm{Al}_{2} \mathrm{O}_{3}$ were applied on a hexagonal plastic cone to suppress reflectance in long wavelengths [11]. The collection efficiencies of the cone for six wavelengths are shown as functions of angles of incidence, with $\sim 50 \%$ reflectance suppression in $600-800 \mathrm{~nm}$ observed. The peaks and valleys found in the curves are due to angular-dependent reflectance caused by interference in the coating.

\subsection{Silicon Photomultipliers}

The use of SiPMs instead of conventional photomultiplier tubes (PMTs) was technically validated by the FACT collaboration [12] and is currently planned by other telescope projects. The focal-plane cameras of the CTA SSTs and MSTs use spherically aligned SiPM arrays, and their prototypes succeeded in Cherenkov observations and Crab detection [13, 14]. Trinity, a wide field-of-view earth-skimming air-shower telescope project, also plans to use SiPMs as photosensors [15-17].

The angular dependence of SiPM PDE can be roughly characterized using Fresnel reflection at the media boundary between air and silicon. However, thin $\mathrm{SiO}_{2}$ and $\mathrm{SiN}$ layers on the surface of silicon substrate causes interference when the SiPM surface is unprotected by thicker $(100-300 \mu \mathrm{m})$ resin coating, resulting in complex angular dependence, as shown in Fig. 2. Also, ROBAST 3 can simulate the interference effect and is promising tool for camera calibration in CR and gamma-ray telescopes.

\footnotetext{
${ }^{3}$ Version 3.2.0 is the latest as of Jul 2021. See https://github. com/ROBAST/ROBAST/tags.

${ }^{4}$ https://pypi.org/project/tmm/
} 


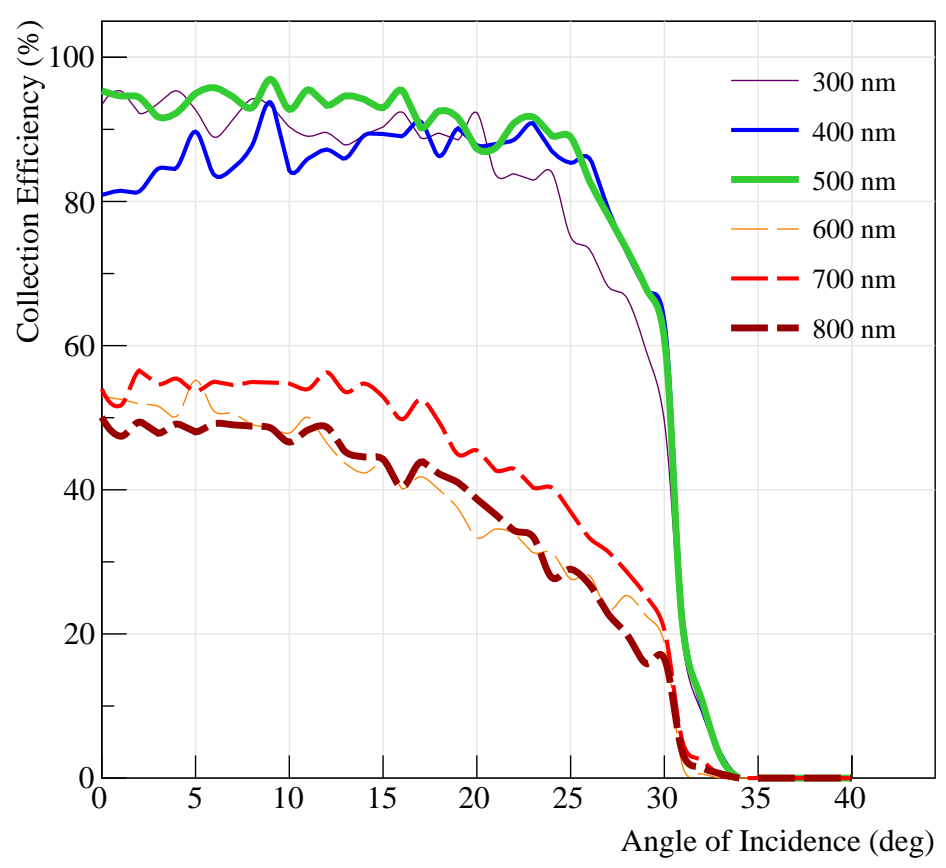

Figure 1: A ROBAST simulation of a hexagonal light concentrator with multilayer coating on the specular surfaces. The solid and dashed curves show the collection efficiency as functions of angles of incidence in UV-to-green and red wavelengths, respectively. The figure was taken from [11] and colorized for this paper.

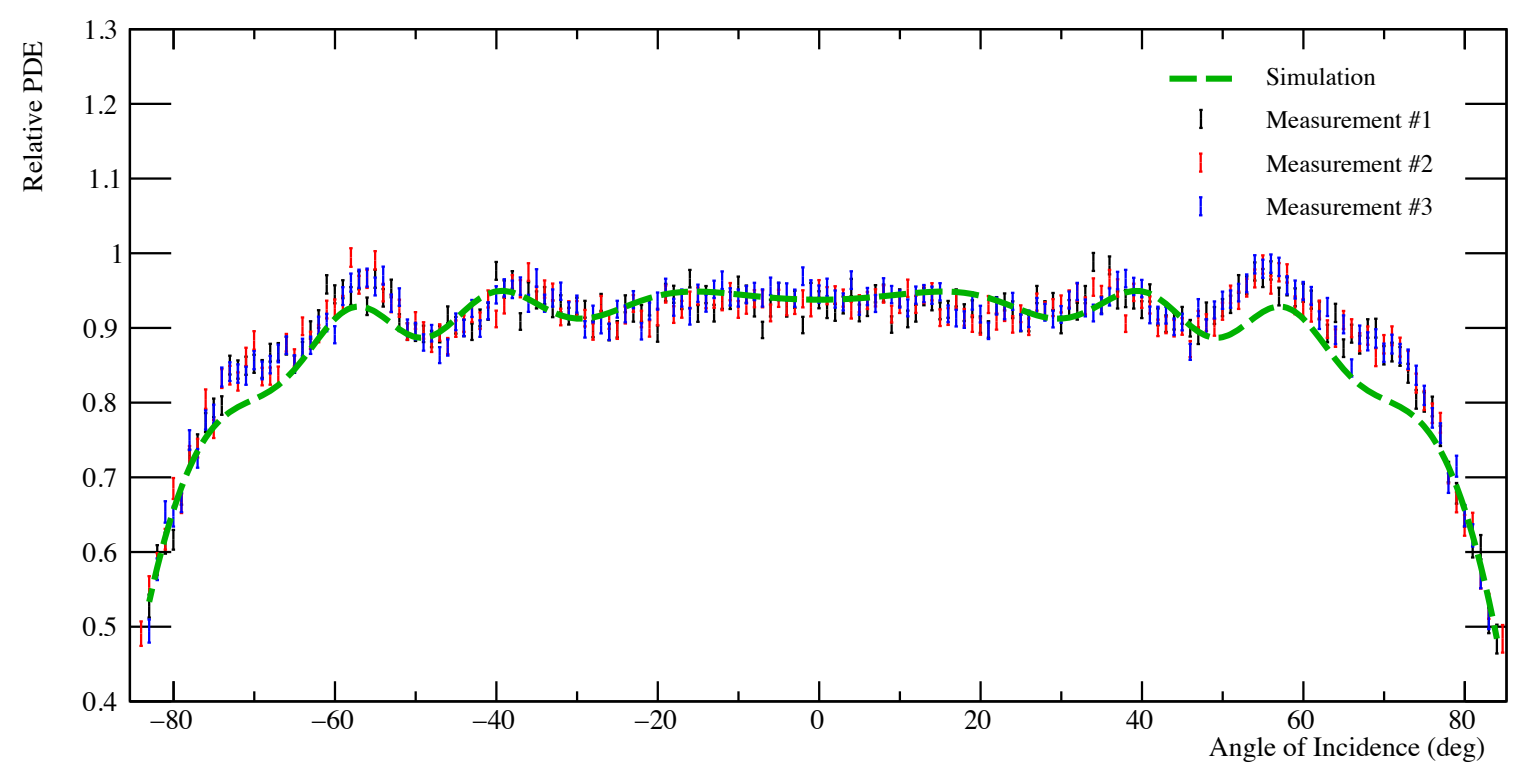

Figure 2: Comparison of measured SiPM PDE at $820 \mathrm{~nm}$ and ROBAST simulation. 
(a)

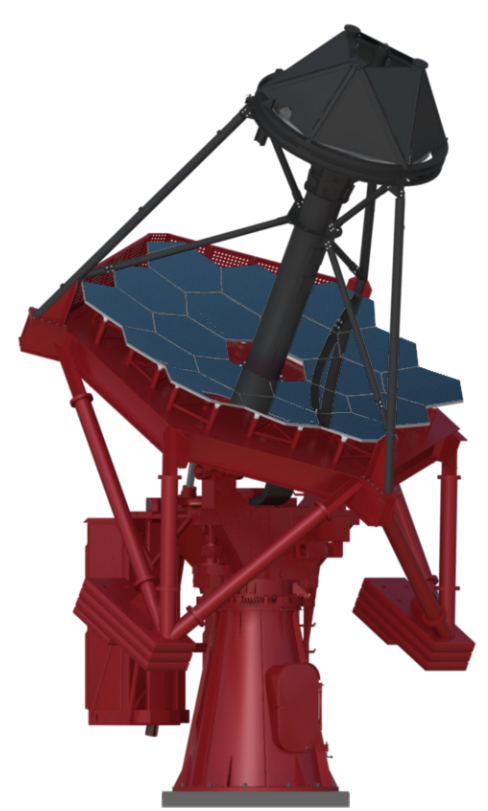

(b)

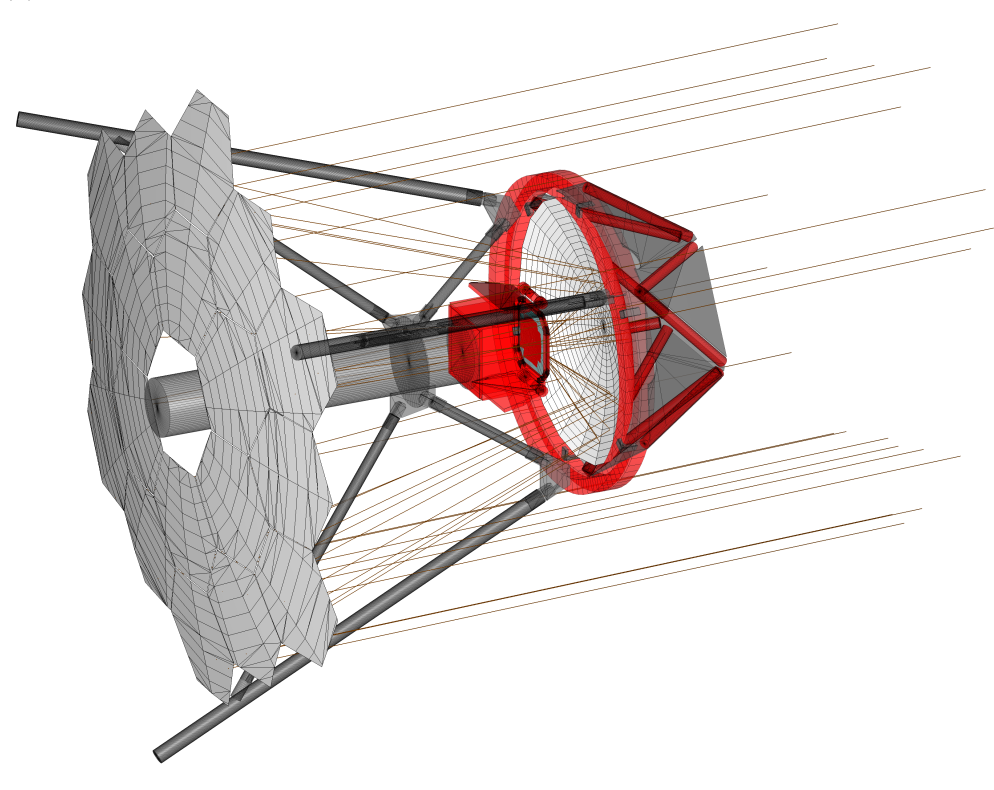

Figure 3: (a) A computer rendering of the CTA SST optical system. Image Credit: G. Pérez, IAC, SMM, via Flickr.com (the Creative Commons Attribution-NonCommercial-NoDerivs 2.0 Generic; CC BY-NC-ND 2.0) (b) An equivalent 3D model of the SST optical system built in the ROBAST framework, where only telescope components that affect the optics performance are simulated. The original 3D CAD file was provided to the author from the CTA SST optics team.

\section{Recent Applications}

ROBAST is the most heavily used in CTA for their optics simulations. Figs. 3(a) and 3(b) compare a 3D model of the CTA SST optical system [18] and its equivalent ROBAST model, manually converted. The complex telescope beams and the secondary mirror system that partly obscure incident photons are modeled using ROBAST, enabling us to calculate the effective area and vignetting accurately.

After the successful use of ROBAST in CTA, various future telescope projects use it for optics simulations [19-21]. Figs. 4(a) and 4(b) shows examples of ROBAST being applied to the Trinity and CRAFFT telescopes [22, 23]. Trinity uses a MACHETE-like optical system [24] combined with SiPMs and light concentrators, and CRAFFT uses a Fresnel lens system to realize a less expensive wide field-of-view telescope without mirrors. Both optical systems can be modeled and simulated using ROBAST without adding any new classes dedicated to individual telescopes.

\section{Conclusion}

ROBAST 3, a new major version of non-sequential ray-tracing software for CR telescopes with interference calculations of multilayer coating, has been released. Currently, ROBAST is used in various optical systems and projects using its functionality to simulate aspherical mirrors and 
(a)

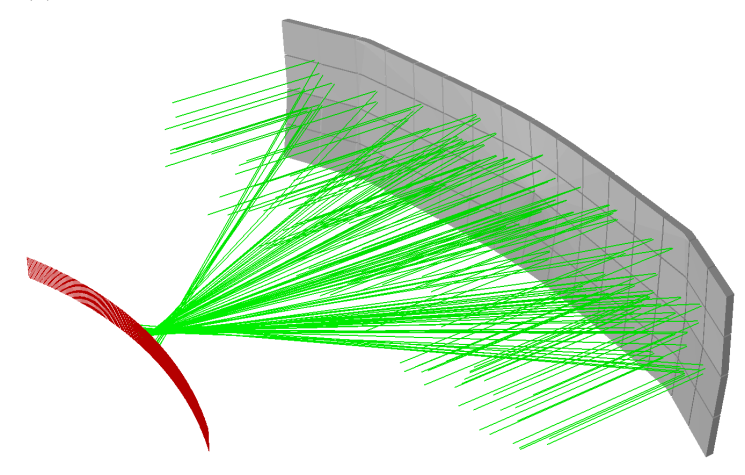

(b)

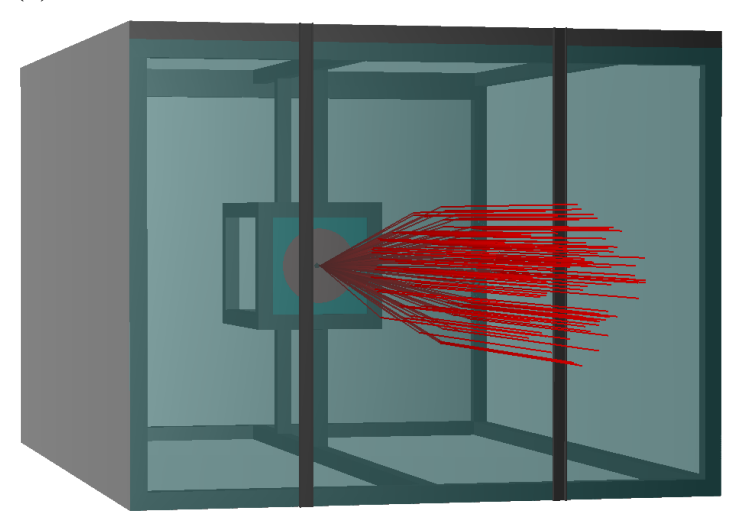

Figure 4: (a) A ROBAST model of a MACHETE-like optical system planned in Trinity. Image credit: The Trinity Collaboration (b) A ROBAST model of the Fresnel lens system and the telescope hut of CRAFFT. Image credit: Yuichiro Tameda

light concentrators. In addition to geometrical optics simulations that were already supported in ROBAST 2, further ROBAST applications with SiPMs and multilayer coating are expected.

\section{References}

[1] A. Okumura, K. Noda and C. Rulten, ROBAST: Development of a ROOT-based ray-tracing library for cosmic-ray telescopes and its applications in the Cherenkov Telescope Array, Astropart. Phys. 76 (2016) 38.

[2] M. Sasaki, A. Kusaka and Y. Asaoka, Design of UHECR telescope with 1 arcmin resolution and $50^{\circ}$ field of view, Nucl. Instr. Meth. Phys. Res. A 492 (2002) 49.

[3] R. Brun, A. Gheata and M. Gheata, The ROOT geometry package, Nucl. Instr. Meth. Phys. Res. A 502 (2003) 676.

[4] A. Okumura, Optimization of the collection efficiency of a hexagonal light collector using quadratic and cubic Bézier curves, Astropart. Phys. 38 (2012) 18.

[5] B. S. Acharya, M. Actis, T. Aghajani, G. Agnetta, J. Aguilar, F. Aharonian et al., Introducing the CTA concept, Astropart. Phys. 43 (2013) 3.

[6] M. Actis, G. Agnetta, F. Aharonian, A. Akhperjanian, J. Aleksić, E. Aliu et al., Design concepts for the Cherenkov Telescope Array CTA: an advanced facility for ground-based high-energy gamma-ray astronomy, Experimental Astronomy 32 (2011) 193.

[7] K. Bernlöhr, Simulation of imaging atmospheric Cherenkov telescopes with CORSIKA and sim_telarray, Astropart. Phys. 30 (2008) 149.

[8] A. N. Otte, D. Garcia, T. Nguyen and D. Purushotham, Characterization of three high efficiency and blue sensitive silicon photomultipliers, Nuclear Instruments and Methods in 
Physics Research Section A: Accelerators, Spectrometers, Detectors and Associated Equipment 846 (2017) 106.

[9] Y. Nakamura, A. Okumura, H. Tajima, N. Yamane and A. Zenin, Characterization of SiPM optical crosstalk and its dependence on the protection-window thickness, in Proceedings of the 5th International Workshop on New Photon-Detectors (PD18), p. 011003, 2019, DOI [https://journals.jps.jp/doi/pdf/10.7566/JPSCP.27.011003].

[10] S. J. Byrnes, Multilayer optical calculations, 2016.

[11] A. Okumura, K. Nishimoto and C. Inoue, "Japanese patent application no. 2021-31974, filed on march 1, 2021."

[12] H. Anderhub, M. Backes, A. Biland, V. Boccone, I. Braun, T. Bretz et al., Design and operation of FACT - the first G-APD Cherenkov telescope, Journal of Instrumentation $\mathbf{8}$ (2013) P06008.

[13] R. White, CHEC: a Compact High Energy Camera for the Cherenkov Telescope Array, Journal of Instrumentation 12 (2017) C12059.

[14] C. Adams, R. Alfaro, G. Ambrosi, M. Ambrosio, C. Aramo, T. Arlen et al., Detection of the Crab Nebula with the 9.7 m prototype Schwarzschild-Couder telescope, Astroparticle Physics 128 (2021) 102562.

[15] A. N. Otte, A. M. Brown, A. D. Falcone, M. Mariotti and I. Taboada, Trinity: An air-shower imaging system for the detection of ultrahigh energy neutrinos, in Proceedings of 36th International Cosmic Ray Conference - PoS(ICRC2019), 2019, DOI [1907. 08732].

[16] A. N. Otte, Studies of an air-shower imaging system for the detection of ultrahigh-energy neutrinos, Phys. Rev. D 99 (2019) 083012.

[17] A. N. Otte, A. M. Brown, M. Doro, A. Falcone, J. Holder, E. Judd et al., Trinity: An air-shower imaging instrument to detect ultrahigh energy neutrinos, 2019.

[18] E. Giro, Canestrari, R., Sironi, G., Antolini, E., Conconi, P., Fermino, C. E. et al., First optical validation of a Schwarzschild Couder telescope: the ASTRI SST-2M Cherenkov telescope, Astron. Astrophys. 608 (2017) A86.

[19] A. Hahn, D. Mazin, P. Bangale, A. Dettlaff, D. Fink, F. Grundner et al., Development of a composite large-size SiPM (assembled matrix) based modular detector cluster for MAGIC, Nuclear Instruments and Methods in Physics Research Section A: Accelerators, Spectrometers, Detectors and Associated Equipment 845 (2017) 89 .

[20] C. Perennes, M. Doro, D. Corti, L. Lessio, M. Mallamaci, M. Mariotti et al., Optical feasibility of an upgrade of the CTA LST camera to SiPM, Nuclear Instruments and Methods in Physics Research Section A: Accelerators, Spectrometers, Detectors and Associated Equipment 984 (2020) 164485. 
[21] Y. Omura, D. R. Bergman, J. F. Krizmanic, K. Nakai and Y. Tsunesada, NICHE detector and operations, Journal of Physics: Conference Series 1468 (2020) 012097.

[22] Y. Tameda, T. Tomida, D. Ikeda, K. Yamazaki, M. Yamamoto, H. Iwakura et al., The status and performance of Cosmic Ray Air Fluorescence Fresnel lens Telescope (CRAFFT) for the next generation uhecr observatory, in Proceedings of 36th International Cosmic Ray Conference - PoS(ICRC2019), vol. 358, p. 435, 2019, DOI.

[23] Y. Tameda, T. Tomida, M. Yamamoto, H. Iwakura, D. Ikeda and K. Yamazaki, Air shower observation by a simple structured Fresnel lens telescope with a single pixel for the next generation of ultra-high-energy cosmic ray observatories, Progress of Theoretical and Experimental Physics 2019 (2019) [https://academic. oup.com/ptep/article-pdf/2019/4/043F01/28533703/ptz025.pdf].

[24] J. Cortina, R. López-Coto and A. Moralejo, MACHETE: A transit imaging atmospheric Cherenkov telescope to survey half of the very high energy $\gamma$-ray sky, Astroparticle Physics 72 (2016) 46 .

\section{Acknowledgments}

We gratefully acknowledge Dr. Steven J. Byrnes, the developer of the Python package tmm, who kindly allowed A. O. to re-implement the package in $\mathrm{C}++$ in the ROBAST library. We also thank the Trinity and CRAFFT collaborations for the excellent ROBAST simulation images. This study was supported by JSPS KAKENHI Grant Numbers JP17H04838, JP18KK0384, and JP20H01916. 